ECONomía Teoría y Práctica • Nueva Época, Número especial, vol.4, agosto 2018, pp. 103-124, http://dx.doi.org/10.24275/ETYPUAM/NE/E042018/Rodriguez

\title{
Impacto de los choques del precio del petróleo en el tipo de cambio y la inflación de México*
}

\section{The Impact of Oil Price Shocks on Mexico's Real Exchange Rate and Inflation}

\section{Domingo Rodríguez Benavides** e Ignacio Perrotini Hernández***}

\begin{abstract}
RESUMEN
Analizamos el impacto dinámico de choques de precios del petróleo en el tipo de cambio real y la inflación de México durante enero 1996-noviembre 2016. Utilizamos un modelo de vectores autorregresivos para modelar procesos posiblemente integrados y un Markov Switching VAR (MS-VAR) con dos estados para examinar el impacto de esos choques en la inflación y el tipo de cambio real con cambio de régimen. Las pruebas de causalidad Granger robustas sugieren que el precio internacional del petróleo en términos reales se determina exógenamente con respecto a las variables domésticas analizadas. Los resultados del MS-VAR revelan que la inflación sólo es afectada por las variaciones del tipo de cambio real y no por las variaciones del precio del petróleo en ambos estados estimados, mientras que las variaciones del precio del petróleo inciden en el tipo de cambio real únicamente en un estado y, por esta vía, posiblemente en la inflación.
\end{abstract}

Palabras clave: Choques del precio del petróleo, impacto asimétrico, México.

Clasificación JEL: C40, F62, Q43.

\begin{abstract}
In this paper the dynamic impact of international oil price shocks on Mexico's real exchange rate and inflation rate in the period January 1996-November 2016 is dealt with. To that aim, we use a VAR model to estimate possibly integrated processes and a two-states Markov Switching VAR (MS-VAR) to assess the impact of those shocks on both inflation and the real exchange rate with a regime switching. Our robust Granger causality tests suggest that the real international oil price is exogenously determined vis-à-vis the domestic variables involved in the analysis. Furthermore, from the MS-VAR results we gather that inflation is uniquely affected by real exchange fluctuations -but not so by oil price variations- in the two estimated states, while oil price changes tend to affect the real exchange rate only in one of the estimated states and, through this channel, they possibly alter inflation.
\end{abstract}

Keywords: Oil Price Shocks; Asymmetric Impact, Markov regime-switch var; Mexico. JEL Classification: C40, F62, Q43.

* Fecha de recepción: 10/11/2017. Fecha de aprobación: 04/01/2018

** Universidad Nacional Autónoma de México, Universidad Autónoma Metropolitana. Correo: dorobe@correo.azc.uam.mx. oRCID: 0000-0002-9169-687X.

*** Universidad Nacional Autónoma de México. Correo: iph@unam.mx. oRCID: 0000-00024330-4448. 
104 ECONOMÍA TeORía y PRÁCTICA • Nueva Época, Número especial, vol. 4, agosto 2018

\section{INTRODUCCIÓN}

El petróleo crudo, al igual que muchos otros productos primarios, es un recurso crucial de la producción, insumo básico en una economía. Incluso se ha planteado que una de las principales causas de la desaceleración de la economía mundial de los años 1999-2000 fue el incremento en los precios internacionales del petróleo (Marte y Villanueva, 2007).

Bohi (1989), Bernanke, Gertler y Watson (1997) y Barsky y Kilian (2001) sostienen que las fluctuaciones en los precios del petróleo per se no afectan el desempeño de la actividad económica, sino que esto más bien depende de la respuesta de la política monetaria ante un choque externo de esa naturaleza, ya que se considera que en las economías en desarrollo la política monetaria es endógena y el canal de transmisión del choque exógeno es directo hacia la estructura de costos de producción (Marte y Villanueva, 2007). La tendencia histórica muestra que el mundo parece haber entrado en una era de alta volatilidad del precio de crudo (Ghosh y Kanjilal, 2014a).

De igual forma, Lanteri (2014) destaca que varios trabajos encuentran que el incremento en los precios del petróleo ha afectado negativamente el desempeño de la economía mundial en virtud de que éstos constituyen una de las principales fuentes de las fluctuaciones económicas.

Existen varios canales por los cuales los choques de los precios del petróleo se pueden transmitir a los fundamentales económicos (Ghosh y Kanjilal, 2014a). Brown y Yücel (2002) reconocen seis canales: (i) el efecto de choques por el lado de la oferta, (ii) el efecto transferencia de riqueza, (iii) el efecto balance real, (iv) el efecto inflacionario, (v) el efecto ajuste del sector y (vi) el efecto inesperado. El efecto de choques por el lado de la oferta consiste en el impacto negativo en el producto ocasionado por el incremento de los costos de producción marginales a medida que aumenta el precio del petróleo. El efecto transferencia de riqueza puede deteriorar los términos de intercambio para los países importadores de petróleo debido a la transferencia de riqueza de los países importadores de petróleo a los países exportadores de petróleo. Brown y Yücel (2002) definen el efecto balance real como la situación en la que las autoridades monetarias no aumentan la oferta monetaria para satisfacer la creciente demanda de dinero debido al alza del precio del petróleo, pero sí incrementan la tasa de interés, lo cual puede desalentar el crecimiento económico, en tanto que el efecto inflación se define como el aumento en el precio del petróleo que genera inflación en una economía (Ghosh y Kanjilal, 2014a). Cuando la inflación observada es generada por choques de costos ocasionados por incrementos en el precio del petróleo, una política monetaria contractiva puede deteriorar la producción a largo plazo por la vía de incrementos en la tasa de interés que podrían desalentar 
la inversión. El efecto de ajuste sectorial funciona a través de los efectos de los choques de precios del petróleo en el mercado de trabajo al cambiar los costos de producción relativos en algunas industrias. Finalmente, el efecto inesperado se centra en la incertidumbre sobre el precio del petróleo y su impacto.

La hipótesis de nuestro trabajo es que las variables macroeconómicas domésticas no inciden en el precio internacional del petróleo en términos reales, mientras que el precio internacional del petróleo en términos reales causa en el sentido de Granger al tipo de cambio real y éste al índice de precios al consumidor. Además, los choques del precio internacional del petróleo en términos reales impactan en la inflación de México mediante su efecto en las variaciones del tipo de cambio real.

En el presente artículo, analizamos el impacto dinámico de choques de precios del petróleo en el tipo de cambio real y la inflación de México durante enero 1996-noviembre 2016. Nuestro estudio empírico se basa en un modelo de vectores autorregresivos y un Markov Switching VAR (MS-VAR) con dos estados que nos permiten examinar el impacto de esos choques en la inflación y en el tipo de cambio real con cambio de régimen. Los resultados del MS-VAR muestran que las variaciones del tipo de cambio real afectan a la inflación y que las variaciones del precio del petróleo en ambos estados estimados no influyen en la inflación. Las variaciones del precio del petróleo, a su vez, inciden en el tipo de cambio real únicamente en un estado y, por este canal, posiblemente en la inflación.

Además de esta introducción, el artículo contiene: una revisión sucinta de diversos estudios empíricos que han analizado la relación entre las fluctuaciones del precio del petróleo y las variables macroeconómicas; una tercera parte en la que explicamos los datos y la metodología econométrica utilizada en las estimaciones; el análisis de los resultados de las pruebas econométricas en la cuarta parte y, finalmente, las conclusiones.

\section{REVISIÓN DE LA LITERATURA EMPÍRICA}

Hamilton (1983) sugiere que los movimientos de precios del crudo se convirtieron en un factor vital para todas las recesiones de los Estados Unidos después de la Segunda Guerra Mundial. Desde entonces, la identificación de los vínculos entre el precio del petróleo crudo y los sectores reales de la economía ha sido uno de los principales campos de investigación teórica y empírica. Harrow y Harrison (1984), Loungani (1986), Gisser y Goodwin (1986) y Uri (1996) son algunos de los pioneros en este campo. Hamilton (2003), Cunado y Pérez de Gracia (2005) y Bachmeier (2008) han modelado los precios del crudo y las actividades económicas (demanda agregada, inflación, empleo y crecimiento económico real). 
Shafiee y Topal (2010) pronosticaron los precios mundiales nominales y reales de los combustibles fósiles de 2009 a 2018 mediante una versión del modelo de reversión a la tendencia de largo plazo con saltos. Sus resultados revelan que los precios reales y nominales del petróleo y del gas natural muestran una tendencia creciente y que los precios de los combustibles fósiles permanecen en un "salto" por arriba de su tendencia en los años posteriores al estudio, mostrando una reversión a la tendencia histórica de largo plazo hasta 2018.

Por otro lado, Kesicki (2010) analiza las razones del alza de precios en el periodo 2003-2008 y la compara con las crisis de los precios del petróleo de 1973 y 19791980. Kesicki (2010) destaca que si bien estas crisis fueron causadas en última instancia por las acciones de oferta de los miembros de la Organización de Países Exportadores de Petróleo (OPEP), las tres crisis de precios del petróleo fueron precedidas por una creciente demanda. Otros aspectos que favorecieron un alto precio del petróleo en los tres casos fueron las bajas inversiones en nuevos yacimientos petroleros, como consecuencia de la baja capacidad de reserva y de un dólar estadounidense débil. Adicionalmente a estos factores, el alza de los precios del petróleo durante 2003-2008 se caracterizó por una intensiva utilización de las refinerías a nivel mundial y por las refinerías que no se adaptaron lo suficientemente rápido a la creciente demanda de combustibles más ligeros. De igual forma, incertidumbres geopolíticas en combinación con los riesgos asociados con la comercialización del petróleo condujeron a que el precio del energético alcanzara niveles de tres dígitos. Para Kesicki (2010), la especulación desempeñó un papel limitado y temporal en el alza de precios durante el periodo 2003-2008.

Lanteri (2014) analiza los principales determinantes macroeconómicos de los precios reales del West Texas Intermediate (WTI) y del Brent. Entre las variables propuestas para explicar los precios internacionales del petróleo se encuentran el índice de la producción industrial de las economías avanzadas, la tasa de interés real de corto plazo de Estados Unidos y el tipo de cambio efectivo del dólar de los Estados Unidos. De igual forma, Lanteri (2014) también analiza el impacto de los precios reales del petróleo en el PIB real, el empleo y los precios domésticos de Estados Unidos, Argentina, España y Noruega. La metodología que utiliza en ambos casos es el modelo SVAR con restricciones de corto plazo. Sus resultados para los países importadores de crudo muestran un impacto diferenciado en los precios y en el empleo ante los incrementos en comparación con las reducciones del precio del petróleo.

También, Alom, Ward y Hu (2013) utilizan modelos SVAR para investigar los efectos macroeconómicos de los choques de los precios internacionales del petróleo y de los alimentos en un conjunto de países de Asia y del Pacífico. Sus resultados revelan que las actividades económicas de los países escasos en recursos que se 
especializan en industria de manufactura pesada, como Corea del Sur y Taiwán, son afectadas de manera fuerte por los precios del petróleo. Por el contrario, las actividades económicas de naciones con escasez de petróleo, como Australia y Nueva Zelanda, con diversos recursos minerales distintos al petróleo, no se ven afectadas por los choques de los precios del petróleo. Además, países sin petróleo, pero que se especializan en servicios financieros internacionales, como Singapur y Hong Kong, no se ven afectados por los incrementos en los precios del crudo. De modo similar, países con limitadas reservas de petróleo, como la India, no se ven afectados por los choques de los precios del petróleo, a diferencia de otros, como Tailandia, que poseen una gran cantidad de recursos naturales diferentes al petróleo y son más sensibles a los choques del precio del petróleo. De lo anterior, Alom et al. (2013) concluyen que los efectos de los precios del petróleo y de los alimentos dependen de las características económicas de cada país.

Ghosh y Kanjilal (2014) analizan el impacto dinámico de especificaciones tanto lineales como no lineales de los choques de precios del petróleo sobre los fundamentos macroeconómicos en la India — que es una economía emergente importadora de petróleo- durante marzo de 1991 a enero de 2009. Mediante la prueba de Toda y Yamamoto, y del modelo var con dos estados de Markov, que permite cambiar el régimen de las variables subyacentes y su relación, los autores concluyen que la inflación y las reservas internacionales están fuertemente afectadas por los choques de los precios del petróleo. Su estudio también confirma que el movimiento en el precio del petróleo es exógeno con respecto al movimiento de las variables macroeconómicas de la India, y que el impacto de los movimientos de estos precios es de naturaleza asimétrica, donde los choques negativos de precios tienen un efecto más pronunciado que los positivos.

Ghosha y Kanjilal (2014b) exploran la cointegración no lineal entre el precio internacional del crudo y el mercado bursátil de la India en un marco multivariado para el periodo comprendido entre enero de 2003 y julio de 2011, mediante pruebas de cointegración de umbral que determinan las rupturas estructurales de manera endógena. Las pruebas rechazan cualquier relación de equilibrio a largo plazo entre las variables para toda la gama de datos. Sin embargo, obtienen resultados distintos al aplicar las pruebas de umbral en tres fases. Siendo la fase II - comprendida entre julio de 2007 y diciembre de 2008 — la más volátil, las pruebas sugieren la existencia de cointegración sólo en la fase III. Mientras que las pruebas de Toda y Yamamoto de causalidad en el sentido de Granger revelan que los movimientos del precio del crudo internacional tienen impacto en el mercado bursátil de la India en las fases II y III sin efecto de retroalimentación. Sus resultados también sugieren que el precio global del crudo se determina exógenamente. 
Taghizadeh-Hesary, Yoshino, Hossein Abadi y Farboudmanesh (2016) estiman el impacto de los movimientos del precio del crudo sobre dos variables macroeconómicas, la tasa de crecimiento del PIB y la tasa de inflación del índice de precios al consumidor de dos países desarrollados: Estados Unidos y Japón, y una economía emergente, la República Popular de China, los cuales tienen como principal característica ser grandes consumidores de petróleo. Taghizadeh-Hesary et al. (2016) averiguan si estas economías reaccionan a los movimientos del precio del petróleo. Sus resultados sugieren que el impacto de las fluctuaciones del precio del petróleo es mucho menor para las economías desarrolladas importadoras de petróleo en comparación con la incidencia que tienen en el crecimiento económico de una economía emergente. Las principales razones de este proceso se atribuyen a la sustitución de combustibles (mayor uso de energía nuclear, gas y renovables), una población declinante en Japón, la revolución del shale gas en los Estados Unidos y los objetivos de eficiencia energética sobre las reservas estratégicas de petróleo, mandatados por los gobiernos en las economías desarrolladas. Todos esos factores han hecho más resistentes a las economías desarrolladas ante los choques del precio del petróleo. Por otra parte, el impacto de los movimientos de los precios del petróleo sobre la tasa de inflación de la República Popular de China resultó ser más moderado que en los dos países desarrollados que se examinaron. La principal causa de esto se atribuye a que la República Popular de China experimenta un mayor desplazamiento en su oferta agregada debido a un mayor crecimiento, lo que le permite evitar un incremento considerable en el nivel de precios después de los choques de precios del petróleo.

Abdlaziz et al. (2016) examinan la relación entre los precios del petróleo y los precios de los alimentos en Indonesia utilizando un modelo de rezagos distribuidos no lineal (NARDL). La prueba del límite de cointegración que aplicaron revela evidencia de cointegración entre el precio de los alimentos, la tasa de crecimiento del PIB y el precio del petróleo. De igual forma, el NARDL estimado para el precio del petróleo en moneda local proporciona una fuerte evidencia de cointegración a corto y largo plazo entre los precios de los alimentos y del petróleo cuando éste se incrementa, mientras que no existe esta relación cuando se reduce el precio del petróleo, dado que resultó no significativa. Asimismo, las estimaciones para el incremento del precio del petróleo medido en dólares estadounidenses resultaron significativas en su estudio.

Ahmed y Wadud (2011) estudian el impacto de la incertidumbre del precio del petróleo sobre las actividades macroeconómicas de Malasia. Con tal fin utilizan un VAR estructural (SVAR) con datos mensuales del periodo 1986-2009, además de un modelo EGARCH. Los resultados del modelo EGARCH muestran un importante efecto asimétrico de los choques del precio del petróleo sobre la volatilidad condicional del precio del petróleo. Las funciones dinámicas de impulso-respuesta obtenidas del modelo SVAR muestran un efecto amortiguador prolongado del choque de la volati- 
lidad del precio del petróleo en la producción industrial de Malasia. Ahmed y Wadud (2011) también encuentran que los niveles del índice de precios al consumidor disminuyen con un choque positivo a la incertidumbre del precio del petróleo. Sus estimaciones también revelan que el Banco Central de Malasia adopta una política monetaria expansiva en respuesta a la incertidumbre del precio del petróleo. El análisis de descomposición de la varianza confirma que la volatilidad en el precio del petróleo es el segundo factor más importante para explicar la varianza de la producción industrial después de sus propios choques.

\section{DATOS Y METODOLOGÍA ECONOMÉTRICA}

\section{II.1. Los datos}

La periodicidad de los datos empleados en este estudio es mensual y comprenden el lapso enero de 1996 a noviembre de 2016. Como precio internacional del petróleo elegimos el del West Texas Intermediate (WTI) en dólares por barril, el cual se tomó del sitio web de la Administración de Información Energética (EIA, por sus siglas en inglés) de los Estados Unidos; este indicador se deflactó por el índice de precios al consumidor de los Estados Unidos para obtener el precio internacional real del petróleo $\left(\right.$ pet $\left._{t}\right)$. En tanto que las variables macroeconómicas internas consideradas fueron el tipo de cambio real bilateral con los Estados Unidos $\left(q_{t}\right)$ y la inflación $\left(i n f_{t}\right)$, que se obtuvo mediante la diferencia logarítmica del índice nacional de precios al consumidor (INPC).

\section{II.2. La prueba de raíz unitaria del multiplicador de Lagrange (LM)}

La prueba LM de rupturas estructurales de Lee y Strazicich (2003a, b) se basa en el siguiente proceso generador de datos:

$$
y_{t}=\delta^{\prime} Z_{t}+X_{t}, \quad X_{t}=\beta X_{t-1}+\varepsilon_{t}
$$

En donde $Z_{t}$ es un vector de variables exógenas y $\varepsilon_{t}$ es un término de error con las propiedades convencionales. Lee y Strazicich (2003a, b) sugirieron básicamente dos formas de establecer los componentes en $Z_{t}$ para el modelo planteado en (1). El primero de ellos, denominado A, permite incorporar un cambio en el intercepto, por lo que $Z_{t}=\left[\begin{array}{lll}1 & t & D_{1 t}\end{array}\right]$, o bien, hasta dos cambios en el intercepto 
bajo la hipótesis alternativa, por lo que en este caso $Z_{t}=\left[\begin{array}{llll}1 & t & D_{1 t} & D_{2 t}\end{array}\right]$. En tanto que el segundo modelo, denominado modelo $\mathrm{C}$, permite incorporar una ruptura estructural en el intercepto y en la pendiente, por lo que de esta manera $Z_{t}=\left[\begin{array}{llll}1 & t & D_{1 t} & D T_{1 t}\end{array}\right]$, o hasta dos rupturas estructurales en el intercepto y en la pendiente, $Z_{t}=\left[\begin{array}{llllll}1 & t & D_{1 t} & D T_{1 t} & D_{2 t} & D T_{2 t}\end{array}\right]$. En estos modelos $D_{j t}=1$ para $t \geq T_{B_{j}}+1, j=1,2$ y 0 en otro caso. Aquí, $T_{B_{j}}$ representa la fecha de la ruptura. El término $D_{j t}$ es una variable dummy indicadora de un cambio en la media que ocurre en el tiempo $T_{B}$, mientras que $T$ es el correspondiente cambio en tendencia. Lee y Strazicich (2003 a, b) utilizan la siguiente regresión para obtener el estadístico Lm de prueba de raíz unitaria:

$$
\Delta y_{t}=\delta^{\prime} \Delta Z_{t}+\phi \bar{S}_{t-1}+\mu_{t}
$$

Donde $\bar{S}_{t}=y_{t}-\widehat{\psi}_{x}-Z_{t} \widehat{\delta}_{t}, t=2, \ldots, T$, y $\widehat{\delta}$ son los coeficientes en la regresión de $\Delta y_{t}$ en $\Delta Z_{t}, \widehat{\psi}_{x}$ está dado por $y_{t}-Z_{t} \delta$. La hipótesis nula de raíz unitaria se describe por $\phi=0$ y el estadístico de prueba Lm está dado por $\bar{\tau}=$ estadistico $-t$ que prueba la hipótesis nula, $\phi=0$. La hipótesis nula de una raíz unitaria se prueba contra la hipótesis alternativa de estacionariedad en tendencia. Los valores críticos se tabulan en Lee y Strazicich (2003 a, b) para los casos de una o dos rupturas, respectivamente.

La prueba LM propuesta por Lee y Strazicich (2003 a, b) no sólo determina endógenamente las fechas de las rupturas estructurales, sino que también evita el problema de sesgo y rechazos espurios (Glynn, Perera y Verma, 2007).

\section{II.3. Pruebas de no causalidad en el sentido de Granger robustas}

La prueba de no causalidad en el sentido de Granger es útil para probar relaciones de causalidad en algunas variables. Sin embargo, requiere que las series de tiempo de dichas variables sean estacionarias. Este requisito es crucial debido a que la presencia de una raíz unitaria modifica las propiedades asintóticas de los estimadores. Aún más, en muchos casos es difícil garantizar que todas las series consideradas sean estacionarias en virtud de que es bien conocido que la potencia de las pruebas 
usuales de raíz unitaria es muy baja. Cuando las series de tiempo de las variables poseen una raíz unitaria, los estadísticos de prueba usuales, tales como la prueba $\mathrm{F}$, las pruebas de Wald y las pruebas LR, no tienen una distribución $\chi^{2}$ asintótica bajo la hipótesis nula (Ding, Kim y Park, 2014). Esto implica que no podemos efectuar pruebas de no causalidad de Granger por medio del modelo vaR cuando algunas variables tienen una raíz unitaria. En estas circunstancias es necesario analizar las relaciones de causalidad con series en primeras diferencias, las cuales proveen información más limitada que la contenida en las series en sus niveles. Toda y Phillips (1993) y Toda y Yamamoto (1995) proponen un método de inferencia robusto en el sentido que los estadísticos de prueba en el VAR sigan una distribución $\chi^{2}$ asintótica bajo la hipótesis nula, aun cuando las variables estén integradas o cointegradas con algún orden arbitrario. Ellos proponen truncar el número de rezagos a $p+d$ en un modelo autorregresivo bivariado utilizando las variables en sus niveles, donde $d$ denota el máximo orden de integración que, por ejemplo, dos series de tiempo consideradas, $x_{t}$ y $y_{t}$, puedan tener. En este caso, entonces, el modelo puede expresarse como:

$$
\begin{aligned}
& y_{t}=\alpha_{0}+\sum_{i=1}^{p+d} \alpha_{i} y_{t-i}+\sum_{i=1}^{p+d} \beta_{i} x_{t-i}+\varepsilon_{y, t}, \\
& x_{t}=\phi_{0}+\sum_{i=1}^{p+d} \phi_{i} x_{t-i}+\sum_{i=1}^{p+d} \psi_{i} y_{t-i}+\varepsilon_{x, t},
\end{aligned}
$$

Donde $p$ se elige de acuerdo con alguno de los criterios de truncamiento de rezagos convencionales. Toda y Yamamoto (1995) muestran que las restricciones lineales o no lineales sobre los primeros $p$ coeficientes $(4 \times p)$ pueden probarse mediante la teoría estándar asintótica. Es decir, es posible efectuar la prueba estándar de no causalidad en el sentido de Granger mediante la prueba $F$ o la prueba de Wald ignorando los últimos $d$ rezagos de las variables, aun cuando éstas estén integradas o cointegradas.

\section{II.4. VAR Markov regime-switching (MRS-VAR)}

Un VAR Markov regime-switching (MRS- VAR) se puede definir como:

$$
Y_{t}=\mu_{s(t)}+\beta_{s(t)} Y_{t-1}+\varepsilon_{t}
$$


Donde $s(t)=1,2, \ldots, K$ representa estados o regímenes en el tiempo $t, \mu_{s(t)}$ es un vector $(n \times 1)$ de interceptos comunes que cambian dependiendo del estado en que se encuentre el proceso, $Y_{t}$ es un vector $(n \times 1)$ de variables endógenas estacionarias, $\varepsilon_{t}$ es un vector $(n \times 1)$ de perturbaciones no observadas donde $\varepsilon_{t} \sim N\left(0, \Sigma_{s(t)}\right), t=1,2, \ldots, n . \beta_{s(t)}$ es una matriz $(n \times n)$ de parámetros autorregresivos, los cuales son dependientes del estado del proceso, $s(t)$ es una variable aleatoria,

$$
\sum S(t)=\left(\begin{array}{ll}
\sigma_{1, s(t)}^{2} & \sigma_{12, s(t)} \\
\sigma_{21, s(t)} & \sigma_{2, s(t)}^{2}
\end{array}\right)
$$

La transición de un estado a otro es un fenómeno estocástico que es gobernado por una matriz de probabilidades de transición $P$ de orden $(k \times k)$ definida de la siguiente forma:

$$
P=\left(\begin{array}{ccc}
p_{11} & \cdots & p_{k 1} \\
\vdots & \ddots & \vdots \\
p_{1 k} & \cdots & p_{k k}
\end{array}\right)
$$

$p_{i j}$ en la matriz $P$ representa la probabilidad de transitar del estado $i-e ́ s i m o$ al estado $j$ en el tiempo $t-1$ al tiempo $t$. La forma más simple de tal especificación es considerar a $s(t)$ como la realización de una cadena de Markov de dos estados con

$$
\begin{aligned}
\operatorname{Pr}(s(t) & \left.=i / s(t-1)=j, s(t-2)=k, \ldots, Y_{t-1}, Y_{t-2}, \&\right) \\
& =\operatorname{Pr}(s(t)=i / s(t-1)=j)=p_{i j}
\end{aligned}
$$

Dado que $s(t)$ no se observa directamente, es necesario inferir su operación a través de la conducta observada de $Y_{t}$; los parámetros necesarios para describir en forma completa la ley de probabilidad que gobierna $Y_{t}$ son la varianza de las innovaciones gaussianas $\sigma_{s(t)}^{2}$, los coeficientes autorregresivos $\beta_{s(t)}$, los interceptos $\mu_{s(t)}$ y $P$ la matriz $(k \times k)$ de probabilidades de transición. 
El espacio de parámetros se estima mediante máxima verosimilitud. Considerando $f\left(y_{t} / s_{t}=j, \Theta\right)$ como la función de verosimilitud para el estado $j$ condicional sobre un conjunto de parámetros $(\Theta)$ en el tiempo $t$, la función de verosimilitud logarítmica completa del modelo puede definirse como:

$$
L n L=\sum_{t=1}^{T} \ln \sum_{j=1}^{2} f\left(y_{t} / s_{t}=j ; \theta\right) \operatorname{Pr}\left(s_{t}=j, \theta\right)
$$

Donde $f\left(y_{t} / s_{t}=j ; \theta\right)$ es la función de densidad de la distribución normal condicional sobre la variable aleatoria $s_{t}$ tomando el valor $j$ en el tiempo $t$. $\operatorname{Pr}\left(s_{t}=j, \theta\right)$ es la probabilidad incondicional de que $s_{t}$ tome el valor $j$ (Ghosh y Kanjilal, 2014). Los estimadores de máxima verosimilitud de los parámetros se definen de la siguiente manera (Hamilton, 1994):

$$
\begin{aligned}
& \mu_{j}=\frac{\sum_{t=1}^{T} y_{t} \cdot P\left(s_{t}=j / y_{t} ; \hat{\theta}\right)}{\sum_{t=1}^{T} P\left(s_{t}=j / y_{t} ; \hat{\theta}\right)} j=1,2, \ldots, k \\
& \sigma_{j}^{2}=\frac{\sum_{t=1}^{T}\left(y_{t}-\mu_{j}\right)^{2} \cdot P\left(s_{t}=j / y_{t} ; \hat{\theta}\right)}{\sum_{t=1}^{T} P\left(s_{t}=j / y_{t} ; \hat{\theta}\right)} j=1,2, \ldots, k \\
& \pi_{j}=\frac{1}{T} \sum_{t=1}^{T} P\left(s_{t}=j / y_{t} ; \hat{\theta}\right) j=1,2, \ldots, k \\
& P\left(s_{t}=\frac{j}{y_{t}} ; \theta\right)=\frac{P\left(s_{t}=j, y_{t} ; \theta\right)}{f\left(y_{t}, \theta\right)}=\frac{f\left(\frac{y_{t}}{s_{t}}=j ; \theta\right) P\left(s_{t}=j ; \theta\right)}{f\left(y_{t}, \theta\right)}
\end{aligned}
$$

El conjunto de ecuaciones no lineales anterior se puede resolver con algún algoritmo iterativo. Hamilton (1994) propone comenzar con algunos valores iniciales arbitrarios supuestos en el espacio de parámetros $\theta=\theta^{(0)}$ para calcular la ecuación (13) y, posteriormente, el lado derecho de las ecuaciones (8) a (10) para $\theta^{(0)} \cdot \theta^{(0)}$ generará un nuevo conjunto de valores de los parámetros, digamos $\theta^{(1)}$. Usando $\theta^{(1)}$ 
los estimadores (10) a (13) obtendremos un nuevo conjunto de parámetros $\theta^{(2)}$. El proceso puede continuar de esta manera hasta que la diferencia entre $\theta^{(m+1)}$ y $\theta^{(m)}$ sea más reducida que el criterio de convergencia especificado (Ghosh y Kanjilal, 2014).

\section{RESULTADOS}

Con la finalidad de averiguar si las series en niveles utilizadas en el análisis son estacionarias o no en presencia de posibles rupturas, efectuamos las pruebas de Lee-Strazicich (2003) con dos rupturas y de Lee-Strazicich (2004) con una ruptura. Los resultados de ambas pruebas aplicadas al logaritmo natural de las series utilizadas se presentan en los cuadros 1 y 2 , para los modelos A y C, respectivamente. Los resultados de las pruebas aplicadas al logaritmo natural de las series con el modelo A en ningún caso permiten rechazar la hipótesis nula de la raíz unitaria, mientras que los resultados de las pruebas efectuadas con el modelo $C$ sugieren que el logaritmo natural del INPC es estacionario, y de igual forma confirman la no estacionariedad de las series del precio internacional del petróleo y del tipo de cambio real.

Cuadro 1. Pruebas de raíz unitaria del mínimo LM

(Modelo A con 1 y 2 dos rupturas)

\begin{tabular}{|c|c|c|c|c|}
\hline VARIABLE & $\widehat{T}_{B}$ & ESTADISTIICO & $\hat{D}_{1 t}$ & $\hat{D}_{2 t}$ \\
\hline \multirow[t]{4}{*}{ pet } & 2014:12 & -2.1091 & -0.1623 & \\
\hline & & & $(-1.927)$ & \\
\hline & 2002:12, 2014:11 & -2.4283 & 0.0866 & -0.2071 \\
\hline & & & $(1.038)$ & $(-2.489)$ \\
\hline \multirow[t]{4}{*}{$q_{t}$} & 2014:12 & -1.3242 & -0.0141 & \\
\hline & & & $(-0.553)$ & \\
\hline & 1999:04, 2014:12 & -1.3778 & 0.0132 & -0.0144 \\
\hline & & & (0.523) & $(-0.565)$ \\
\hline \multirow[t]{4}{*}{ inpc $_{t}$} & 2002:02 & -0.2238 & 0.0041 & \\
\hline & & & (1.429) & \\
\hline & 2002:02, 2004:05 & -0.3137 & 0.0042 & 0.0014 \\
\hline & & & $(1.450)$ & (0.519) \\
\hline
\end{tabular}

Nota: pruebas efectuadas con un rezago en Rats 8.1. $\widehat{T}_{B}$ denota los puntos estimados de la ruptura. Los números entre paréntesis corresponden a los estadísticos t. Los valores críticos a los niveles de significancia del 5 y del 10 por ciento para la prueba con una ruptura son -3.566 y -3.211 , mientras que para la prueba con dos rupturas son -3.842 y -3.504 , respectivamente, con $T=100$. 
De lo anterior se puede concluir que el logaritmo natural de las series en sus niveles tiene orden de integración igual a uno. Los resultados de las pruebas de Lee y Strazicich $(2003 \mathrm{a}, \mathrm{b})$ aplicadas a las primeras diferencias de las series del precio internacional del petróleo, del tipo de cambio real y de la inflación se presentan en los cuadros 3 y 4 .

Cuadro 2. Pruebas de raíz unitaria del mínimo LM (Modelo C con 1 y 2 dos rupturas)

\begin{tabular}{|c|c|c|c|c|c|c|}
\hline VARIABLE & $\hat{T}_{B}$ & ESTADISSTICO & $\widehat{D}_{1 t}$ & $\hat{d} t_{1}^{*}$ & $\hat{D}_{2 t}$ & $\widehat{d} t_{2}^{*}$ \\
\hline \multirow[t]{4}{*}{ pet } & 2011:04 & -4.1267 & -0.1258 & 0.0100 & & \\
\hline & & & $(-1.530)$ & $(0.728)$ & & \\
\hline & $2004: 11,2014: 10$ & $-5.7439^{*}$ & -0.1905 & 0.0994 & -0.0068 & -0.1129 \\
\hline & & & $(-2.336)$ & $(4.831)$ & $(-0.084)$ & $(-4.858)$ \\
\hline \multirow{4}{*}{$q_{t}$} & 1998:12 & -2.6952 & 0.0494 & -0.0210 & & \\
\hline & & & (2.015) & $(-3.730)$ & & \\
\hline & 1999:07, 2013:09 & -4.4849 & 0.0158 & -0.0135 & -0.0194 & 0.0009 \\
\hline & & & $(0.651)$ & $(-2.842)$ & $(-0.791)$ & (0.194) \\
\hline \multirow[t]{4}{*}{$i n p c_{t}$} & 1999:01 & $-4.0379 * *$ & 0.0029 & -0.0085 & & \\
\hline & & & $(1.032)$ & $(-12.157)$ & & \\
\hline & 1999:01, 2003:07 & $-6.4472^{* * *}$ & 0.0007 & -0.0072 & -0.0004 & -0.0028 \\
\hline & & & $(0.250)$ & $(-10.516)$ & $(-0.138)$ & $(-6.576)$ \\
\hline
\end{tabular}

Nota: pruebas efectuadas con un rezago en Rats 8.1. $\widehat{T}_{B}$ denota los puntos estimados de la ruptura. *, $\star *, * * *$ denotan significancia del estadístico de prueba a los niveles de 10, 5 y 1 por ciento, respectivamente. Los valores críticos de las pruebas del modelo $\mathrm{C}$ se contrastaron de los cuadros de Lee y Strazicich (2003), Lee y Strazicich (2004) y Rodríguez (2009).

Cuadro 3. Pruebas de raíz unitaria del mínimo LM

(Modelo A con 1 y 2 dos rupturas)

\begin{tabular}{ccccc}
\hline \multirow{2}{*}{ VARIABLE } & $\widehat{T}_{B}$ & ESTADísTICO & $\widehat{D}_{1 t}$ & $\widehat{D}_{2 t}$ \\
\hline \multirow{2}{*}{ dpet } & $2014: 06$ & $-4.4568^{* * *}$ & -0.0530 & \\
& & $(-0.624)$ & \\
& $2001: 11,2014: 06$ & $-11.5588^{* * *}$ & 0.0148 & -0.0524 \\
& & $(0.175)$ & $(-0.621)$
\end{tabular}


116 ECONOMÍA TeOrÍA y PrÁCTICA • Nueva Época, Número especial, vol. 4, agosto 2018

\section{Cuadro 3. Continuación}

\begin{tabular}{|c|c|c|c|c|}
\hline VARIABLE & $\hat{T}_{B}$ & ESTADISTIICO & $\hat{D}_{1 t}$ & $\hat{D}_{2 t}$ \\
\hline \multirow[t]{4}{*}{$d q_{t}$} & 2014:11 & $-9.9422^{* * *}$ & 0.0649 & \\
\hline & & & $(2.531)$ & \\
\hline & $2003: 02,2008: 10$ & $-10.5803^{* * *}$ & -0.0381 & 0.0027 \\
\hline & & & $(-1.512)$ & $(0.107)$ \\
\hline \multirow[t]{4}{*}{$\inf t_{t}$} & 1998:12 & -2.9102 & -0.0072 & \\
\hline & & & $(-2.25)$ & \\
\hline & $1999: 12,2007: 04$ & -3.2963 & -0.0018 & 0.0026 \\
\hline & & & $(-0.614)$ & (0.923) \\
\hline
\end{tabular}

Nota: pruebas efectuadas con un rezago en Rats 8.1. $\widehat{T}_{B}$ denota los puntos estimados de la ruptura. Los números entre paréntesis corresponden a los estadísticos t. Los valores críticos a los niveles de significancia del 5 y del 10 por ciento para la prueba con una ruptura son -3.566 y -3.211 , mientras que para la prueba con dos rupturas son -3.842 y -3.504 , respectivamente.

\section{Cuadro 4. Pruebas de raíz unitaria del mínimo LM (Modelo C con 1 y 2 dos rupturas)}

\begin{tabular}{|c|c|c|c|c|c|c|}
\hline VARIABLE & $\widehat{T}_{B}$ & ESTADISTIICO & $\hat{D}_{1 t}$ & $\widehat{d} t_{1}^{*}$ & $\hat{D}_{2 t}$ & $\hat{d} t_{2}^{*}$ \\
\hline \multirow[t]{4}{*}{ dpet } & $1998: 12$ & $-6.4220^{* * *}$ & -0.2735 & 0.1658 & & \\
\hline & & & $(-3.072)$ & $(5.447)$ & & \\
\hline & $2008: 06,2010: 07$ & $-12.0072^{* * *}$ & -0.1612 & 0.0305 & -0.0561 & -0.0054 \\
\hline & & & $(-1.899)$ & $(1.647)$ & $(-0.670)$ & $(-0.278)$ \\
\hline \multirow[t]{4}{*}{$d q_{t}$} & $2008: 12$ & $-11.2614^{* * *}$ & 0.0514 & -0.0212 & & \\
\hline & & & $(2.050)$ & $(-5.587)$ & & \\
\hline & $2008: 07,2011: 06$ & $-11.8286^{* * *}$ & 0.0762 & -0.0113 & 0.0897 & -0.0197 \\
\hline & & & (3.129) & $(-2.425)$ & (3.684) & $(-3.630)$ \\
\hline \multirow[t]{4}{*}{$\inf t_{t}$} & $1998: 12$ & $-4.4297^{*}$ & -0.0008 & 0.0005 & & \\
\hline & & & $(-0.2738)$ & (1.078) & & \\
\hline & 1999:01, 2001:08 & $-7.3823^{* * *}$ & -0.0137 & 0.0092 & 0.0069 & -0.0025 \\
\hline & & & $(-5.059)$ & (7.081) & (2.674) & $(-4.402)$ \\
\hline
\end{tabular}

Nota: pruebas efectuadas con un rezago en Rats 8.1. $\widehat{T}_{B}$ denota los puntos estimados de la ruptura. ${ }^{*}$ $* *, * * *$ denotan significancia del estadístico de prueba a los niveles de 10,5 y 1 por ciento, respectivamente. Los valores críticos de las pruebas del modelo C se contrastaron de los cuadros de Lee y Strazicich (2003), Lee y Strazicich (2004) y Rodríguez (2009). 
De los cuadros 3 y 4 se infiere que, para las primeras diferencias de los logaritmos naturales de las series del precio internacional del petróleo en términos reales, del tipo de cambio real, así como de la inflación, se rechaza la hipótesis nula de raíz unitaria cuando se incorporan dos rupturas en el modelo $\mathrm{C}$, incluso al nivel de significancia del 1 por ciento. A partir de los resultados de las pruebas de raíz unitaria con rupturas estructurales concluimos que las primeras diferencias de los logaritmos naturales de las series consideradas en el análisis son estacionarias.

En el cuadro 5 se muestran los resultados de las pruebas de no-causalidad en el sentido de Granger efectuadas a las series en sus niveles a partir de un VAR con siete rezagos. ${ }^{1}$ De dichas pruebas es posible inferir que ninguna de las variables macroeconómicas domésticas consideradas tiene influencia en el precio internacional del petróleo. En cambio, el precio internacional en términos reales únicamente tiene incidencia en el tipo de cambio real al igual que el INPC.

Cuadro 5. Resultados de las pruebas de No-Causalidad de Granger

\begin{tabular}{|l|c|c|c|}
\hline \multicolumn{4}{|c|}{ VARIABLE DEPENDIENTE pet } \\
\hline Excluida & $\chi(2)$ & g.l. & Valor-p \\
\hline$q$ & 6.2583 & 7 & {$[0.5099]$} \\
\hline inpc & 4.8347 & 7 & {$[0.6801]$} \\
\hline Ambas & 10.8532 & 14 & {$[0.6975]$} \\
\hline
\end{tabular}

\begin{tabular}{|l|c|c|c|}
\hline \multicolumn{4}{|c|}{ VARIABLE DEPENDIENTE $q$} \\
\hline Excluida & $\chi(2)$ & g.l. & Valor-p \\
\hline pet & 26.9105 & 7 & {$[0.0003]$} \\
\hline inpc & 17.6346 & 7 & {$[0.0137]$} \\
\hline Ambas & 46.0041 & 14 & {$[0.0000]$} \\
\hline
\end{tabular}

\begin{tabular}{|l|c|c|c|}
\hline \multicolumn{4}{|c|}{ VARIABLE DEPENDIENTE inpc } \\
\hline Excluida & $\chi(2)$ & g.l. & Valor-p \\
\hline pet & 3.5389 & 7 & {$[0.8311]$} \\
\hline$q$ & 10.4419 & 7 & {$[0.1649]$} \\
\hline Ambas & 11.7597 & 14 & {$[0.6256]$} \\
\hline
\end{tabular}

${ }^{1}$ Incorporamos un número grande de rezagos en el VAR, porque el modelo supera en su mayoría las pruebas LM de autocorrelación sólo hasta con siete rezagos. Esto es muy posible que se deba a que no se están considerando algunos términos determinísticos, tales como variables dummy, que posiblemente sean requeridos en el modelo. No obstante, para el propósito del modelo utilizado para estas series, estos términos no se requieren. 
Por otro lado, ninguna de las macrovariables domésticas causa en el sentido de Granger al precio internacional del petróleo en términos reales, lo cual, como era de suponerse, verifica la exogeneidad estricta del precio internacional en términos reales del precio del petróleo.

Con el fin de determinar algún posible impacto no lineal del precio internacional del petróleo en las variables macroeconómicas de México, estimamos un Markov Switching VAR con las variables en primeras diferencias del precio petróleo, del tipo de cambio real y de la inflación.

Los resultados de la estimación del MS-VAR con dos estados para las variables $d p e t, d q$ e inf se muestran en los cuadros 6 y 7 . El modelo se estimó con un rezago, en cuya determinación coincidieron todos los criterios informativos utilizados en la determinación de rezagos en el VAR. Los estados para los choques del petróleo se definieron como regímenes crecientes y decrecientes del precio del petróleo. El cuadro 6 presenta los resultados para el estado o régimen $1, s(t)=1$. Como se esperaba, al igual que las variables en sus niveles, las variables macroeconómicas domésticas en sus primeras diferencias no tienen impacto en las variaciones del precio internacional del petróleo en términos reales, dado que éstas únicamente son afectadas por su propio valor rezagado. Algo similar ocurre con las fluctuaciones del tipo de cambio real, dado que sólo su propio valor rezagado resultó significativo, mientras que para la inflación interna únicamente tienen incidencia los rezagos del tipo de cambio real y de la misma inflación.

Con respecto al régimen 2, $s(t)=2$, cuyos resultados se muestran en el cuadro 7 , tanto las variables macroeconómicas domésticas como el propio valor rezagado de las variaciones del precio internacional del petróleo en términos reales no tienen impacto alguno en estas últimas. Un resultado similar ocurre con las variaciones del tipo de cambio real, que sólo se ven afectadas por su propio rezago en este estado. La única variable que mostró coeficientes significativos fue la inflación, que se vio afectada por las variaciones del tipo de cambio real rezagadas y por la misma inflación desfasada un periodo.

El cuadro 8 presenta la matriz de probabilidades de transición. Como se puede ver en ella, el régimen 1 es más persistente. La probabilidad de que el estado 1 sea seguido por un mes del estado 2 es de 0.86 . De igual forma, el régimen 1 persistirá por aproximadamente siete meses, mientras que el sistema analizado permanecerá en el régimen 2 cerca de tres meses.

Por último, la gráfica 1 muestra la probabilidad condicional de los regímenes 1 y 2. En ella se observa que la probabilidad del régimen 1 es la que predomina en comparación con el régimen 2 a lo largo de toda la muestra. 
Cuadro 6. Resultados del vaR Markov regime-switching

\begin{tabular}{lccc}
\hline ESTADO 1 & dpet & $d q$ & inf \\
\hline $\operatorname{dpet}(-1)$ & $0.80^{*}$ & $-0.14^{*}$ & 0.01 \\
& {$[0.00]$} & {$[0.00]$} & {$[0.63]$} \\
$d q(-1)$ & -0.21 & $0.40^{*}$ & $0.16^{*}$ \\
& {$[0.74]$} & & {$[0.00]$} \\
$\inf (-1)$ & 0.93 & 0.21 & $0.68^{*}$ \\
& {$[0.27]$} & {$[0.36]$} & {$[0.00]$}
\end{tabular}

Nota: * implica significancia al nivel del 5 por ciento. Los números entre corchetes son el valor- $p$ asociado a cada parámetro estimado.

Cuadro 7. Resultados del vaR Markov regime-switching

\begin{tabular}{lccc}
\hline ESTADO2 & $d$ pet & $d q$ & inf \\
\hline $\operatorname{dpet}(-1)$ & 0.13 & 0.03 & 0.00 \\
& {$[0.11]$} & {$[0.17]$} & {$[0.18]$} \\
$d q(-1)$ & 0.28 & $0.21^{*}$ & $0.05^{*}$ \\
$\inf (-1)$ & {$[0.40]$} & {$[0.00]$} & {$[0.00]$} \\
& -0.10 & -0.12 & $0.57^{*}$ \\
& {$[0.93]$} & {$[0.64]$} & {$[0.00]$} \\
\hline
\end{tabular}

Nota: * implica significancia al nivel del 5 por ciento. Los números entre corchetes son el valor-p asociado a cada parámetro estimado. 
120 ECONOMía Teoría y PRÁCTICA • Nueva Época, Número especial, vol. 4, agosto 2018

Cuadro 8. Matriz de probabilidad de transición

\begin{tabular}{ccc}
\hline ESTADO & 1 & 2 \\
\hline 1 & 0.63 & 0.14 \\
2 & 0.37 & 0.86 \\
\hline
\end{tabular}

Cuadro 9. Duración esperada de cada estado

\begin{tabular}{cc}
\hline ESTADO & $Y$ \\
\hline Estado 1 & 2.73 meses \\
Estado 2 & 7.27 meses \\
\hline
\end{tabular}

Gráfica 1. Probabilidades condicionales del sistema de encontrarse en los regímenes 1 y 2

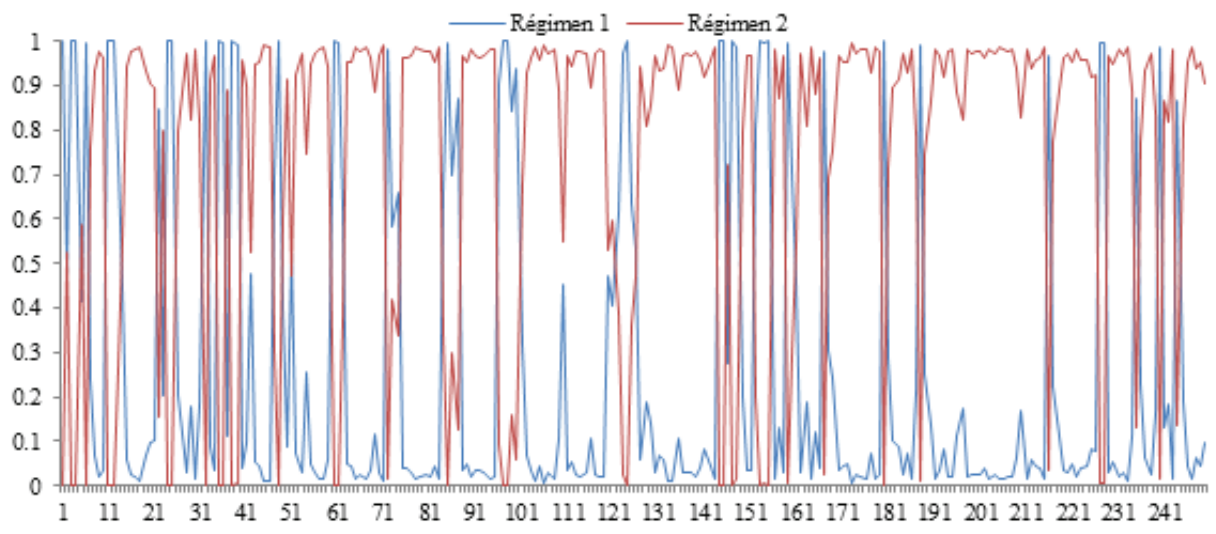

\section{Conclusiones}

En este trabajo analizamos el impacto dinámico lineal y no lineal de los choques del precio internacional del petróleo en términos reales sobre algunas variables macroeconómicas de México en el periodo enero de 1996-noviembre de 2016. Con tal fin, usamos el modelo var extendido propuesto por Toda y Yamamoto, el cual se puede utilizar independientemente del orden de integración de las variables analizadas y de si se encuentran cointegradas o no.

Los resultados de la prueba de Toda y Yamamoto, aplicada a un VAR extendido con las variables precio internacional del petróleo en términos reales, el West Texas Intermediate, el tipo de cambio real y el índice nacional de precios al consumidor, 
revelaron que ninguna de las variables macroeconómicas consideradas tiene incidencia en el precio internacional del petróleo en términos reales. En tanto que al tipo de cambio real únicamente lo causa en el sentido de Granger el precio internacional del petróleo en términos reales, y al índice de precios al consumidor lo causa en el sentido de Granger sólo el tipo de cambio real.

Los resultados del Markov Switching VAR (MS-VAR), estimado con dos estados, sugieren que el régimen 2 es más persistente que el régimen 1 , dado que el régimen 2 perdura por más de siete meses en comparación con el estado 1, que se mantiene aproximadamente tres meses. En el régimen 1, los choques del precio internacional del petróleo en términos reales tienen impacto sobre las fluctuaciones del tipo de cambio real, pero no sobre la inflación, la cual únicamente se ve afectada por las variaciones del tipo de cambio real. Así, encontramos que la forma en la cual los choques del precio internacional del petróleo en términos reales impactan en la inflación de México es mediante su efecto en las variaciones en el tipo de cambio real.

\section{REFERENCIAS BIBLIOGRÁFICAS}

Ahmed, Huson J. y Wadud, I. K. M. (2011), "Role of oil price shocks on macroeconomic activities: an SVAR approach to the Malaysian economy and monetary responses", Energy Policy, 39, 8062-9. DoI: 10.1016/j. enpol.2011.09.067

Alom, Fardous; Ward, Bert. D. y Hu, Baiding (2013), "Macroeconomic effects of world oil and food price shocks in Asia and Pacific economies: application of SVAR models", Opec Energy Review, september, 37 (3), pp. 327 372.

Amisano, Gianni y Giannini, Carlo (1997), Topics in Structural VAR Econometrics, Springer-Verlag, Berlin.

Bachmeier, Lance (2008), "Monetary policy and the transmission of oil shocks", Journal of Macroeconomics, 30, pp. 1738-1755.

Brown, Stephen P. A. y Yücel, Milne K. (2002), "Energy prices and aggregate economic activity: An interpretative survey", Quarterly Review of Economics and Finance, 42, pp. 193-208.

Burbidge, John y Harrison, Alan (1984), "Testing for the Effects of Oil-Price Rises Using Vector Autoregressions", International Economic Review, 25, pp. 459-484.

Cunado, Juncal y Pérez de Gracia, Fernando (2005), "Oil prices, economic activity and inflation: evidence for some Asian countries", The Quarterly Review of Economics and Finance, 45 (1), pp. 65-83. 
122 ECONOMÍA TeOrÍA y PRÁCTICA • Nueva Época, Número especial, vol. 4, agosto 2018

Ding, Haoyuan; Kim, Hyung-Gun y Park, Sung Y. (2014), "Do net positions in the futures market cause spot prices of crude oil?", Economic Modelling, 41, pp. 177-90.

Glynn, John; Perera, Nelson y Verma, Reetu (2007), "Unit root tests and structural breaks: A survey with Applications", Revista de Métodos Cuantitativos para la Economía y la Empresa, 3, pp. 63-79.

Ghosh, Sajal y Kanjilal, Kakali (2014a), "Oil price shocks on Indian economy: evidence from Toda Yamamoto and Markov regime-switching VAR", Macroeconomics and Finance in Emerging Market Economies, 7 (1), pp. $122-139$.

(2014b), "Co-movement of international crude oil price and Indian stock market: evidences from nonlinear cointegration tests", Energy Economics, 53, pp. 111-117, http://dx.doi.org/10.1016/j.eneco.2014.11.002

Gisser, Micha y Goodwin, Thomas H. (1986), "Crude oil and the macroeconomy: tests of some popular notions", Journal of Money, Credit and Banking, 18 (1), pp. 95-103.

Hamilton, James D. (1983), "Oil and the macroeconomy since World War II", Journal of Political Economy, 91 (2), pp. 228-248. (1994), Time series analysis, Princeton, NJ: Princeton University Press. (2003). "What is an oil shock?", Journal of Econometrics, 113 (2), pp. 363-398.

Jarque Carlos M. y Bera, Anil K. (1987), “A Test for Normality of Observations and Regression Residuals", International Statistical Review, 55, pp. 163172.

Kesicki, Fabian (2010), “The third oil price surge - What's different this time?", Energy Policy, 38 (3), pp. 1596-606.

Lanteri, Luis N. (2014), "Determinantes de los precios reales del petróleo y su impacto sobre las principales variables macroeconómicas: EU, España, Noruega y Argentina", Economía: Teoría y Práctica, 41, pp. 45-70.

Lee, Junsoo y Strazicich, Mark C. (2003a), "Minimum LM unit root test with one structural break", Mimeo. (2003b), "Minimum Lagrange multiplier unit root test with two structural breaks", The Review of Economics and Statistics, 85, pp. 1082-1089.

Loungani, Prakash (1986), "Oil price shocks and the dispersion hypothesis", The Review of Economics and Statistics, 68, pp. 536-539.

Lütkepohl, Helmut y Krätzig, Markus (2004), Applied Time Series Econometrics, Cambridge University Press, Cambridge. 
Marte, Odalis y Villanueva, Brenda (2007), "Los precios internacionales del petróleo, el PIB real y los precios de la economía dominicana", Ciencia y Sociedad, 32 (2), pp. 190-216.

Pfaff, Bernhard (2008), Analysis of Integrated and Cointegrated Time Series with $R$, Springer. (2008b), "Var, Svar and Svec Models: Implementation within R Package Vars", Journal of Statistical Software, 27 (4), pp. 1-32.

Perlin, Marcelo (2010), "Ms Regress - The MATLAB Package for Markov Regime Switching Models", http://ssrn.com/abstract=1714016 (Consultado en febrero de 2017).

Shafiee, Shahriar y Topal, Erkan (2010), “A long-term view of worldwide fossil fuel prices”, Applied Energy, 87 (3), pp. 988-1000.

Uri, Noel D. (1996), "Changing crude oil price effects on US agricultural employment", Energy Economics, 18, pp. 185-202.

Tang, Weiqi; Wu, Libo y Zhang, ZhongXiang (2010), "Oil price shocks and their short- and long-term effects on the Chinese economy", Energy Economics, 32, S3-S14.

Taghizadeh-Hesary, F.; Yoshino, Naoyuki; Majid Mohammadi Hossein Abadi y Farboudmanesh, Rosa (2016), "Response of macro variables of emerging and developed oil importers to oil price movements", Journal of the Asia Pacific Economy, 21 (1), pp. 91-102. 
